\title{
A percepção de pessoas surdas sobre o acolhimento e cuidado dos profissionais de enfermagem em unidades de emergência
}

\author{
The perception of deaf people about the reception and care of nursing professionals in \\ emergency units
}

\section{La percepción de las personas sordas sobre la recepción y atención de profesionales de enfermería en unidades de emergencia}

Daniella Gomes de Oliveira Costa ${ }^{1}$, Luzia Lima dos Santos ${ }^{2}$, Beverly Scardini Menegazzo Nunes ${ }^{3}$, Luna Vitória Cajé Moura ${ }^{3}$, Leonardo Santos Silva ${ }^{3}$, Raiane Silva Santos ${ }^{3 *}$.

\section{RESUMO}

Objetivo: Compreender a percepção de pessoas surdas sobre o acolhimento e cuidado dos profissionais de enfermagem em unidades de emergência. Métodos: Estudo de pesquisa exploratória, com abordagem qualiquantitativa, por meio de uma entrevista realizada com dez surdos, presencialmente e/ou virtualmente, utilizando a linguagem brasileira de sinais (LIBRAS), com questões caracterizadoras do perfil dos participantes, seguidas por questões abertas abordando o conhecimento conceitual sobre acolhimento, cuidado e experiências vividas na emergência pelo surdo e a visão do mesmo sobre os profissionais de enfermagem. Resultados: Denota-se a partir dos relatos, ou da falta deles, que os participantes pouco sabem sobre saúde e seus direitos sobre ela, assim como desconhecem o que realmente significa acolhimento e cuidado partindo de um profissional enfermeiro. Além disso, todos ressaltaram a importância do bom uso da comunicação como fator primordial para uma assistência qualificada. Conclusão: Diante do exposto percebeu-se a urgência do fazer saúde com mais inclusão para o paciente surdo. De acordo com relatos dos vários indivíduos, existe pouco ou nenhum domínio da LIBRAS por parte dos profissionais de enfermagem, dificultando $o$ atendimento holístico para pessoas com surdez na emergência hospitalar.

Palavras-chave: Surdez, Comunicação, Acolhimento, Enfermagem, Emergência.

\begin{abstract}
Objective: To understand the perception of deaf people about the reception and care of nursing professionals in emergency units. Methods: Exploratory research study, with a quantitative quali approach, through an interview conducted with ten deaf people, in person and / or virtually, using a Brazilian sign language (LIBRAS), with questions that characterize the profile of the participants, followed by open questions addressing the conceptual knowledge about reception, care and experiences lived in the emergency by the deaf and the vision of the same about nursing professionals. Results: It is evident from the reports, or the lack of them, that the participants know little about health and their rights over it, as well as they do not know what welcoming and care really means from a professional nurse. In addition, all stressed the importance of the good use of communication as a primary factor for qualified assistance. Conclusion: Given the above, there is an urgent need to do health with more inclusion for the deaf patient. According to reports of the various requirements, there is little or no domain of LIBRAS on the part of nursing professionals, making holistic care for people with deafness in the hospital emergency difficult.
\end{abstract}

Key words: Deafness, Communication, Reception, Nursing, Emergencies.

\footnotetext{
${ }^{1}$ Hospital 5 de Outubro, Canaã dos Carajás - PA.

2 Hospital Adventista Silvestre, Rio de Janeiro - RJ.

${ }^{3}$ Faculdade Adventista da Bahia (FADBA), Cachoeira - BA. *E-mail: rribeiro.rs73@gmail.com
} 


\section{RESUMEN}

Objetivo: Comprender la percepción de las personas sordas sobre la recepción y atención de los profesionales de enfermería en las unidades de emergencia. Métodos: Estudio de investigación exploratorio, con enfoque cuali cuantitativo, a través de una entrevista realizada a diez personas sordas, en persona y / o virtualmente, utilizando la lengua de signos brasileña (LIBRAS), con preguntas que caracterizan el perfil de los participantes, seguido de preguntas abiertas. abordar el conocimiento conceptual sobre la acogida, el cuidado y las vivencias vividas en la emergencia por los sordos y la visión de los mismos sobre los profesionales de enfermería. Resultados: Es evidente por los informes, o la falta de ellos, que los participantes saben poco sobre la salud y sus derechos sobre ella, así como tampoco saben lo que realmente significa la acogida y el cuidado de una enfermera profesional. Además, todos destacaron la importancia del buen uso de la comunicación como factor primordial para la asistencia calificada. Conclusión: Ante lo anterior, se percibió la urgencia de hacer salud con más inclusión para el paciente sordo. Según los informes de las distintas personas, el dominio de LIBRAS por parte de los profesionales de enfermería es escaso o nulo, lo que dificulta la atención integral de las personas con sordera en la emergencia hospitalaria.

Palabras clave: Sordera, Comunicación, Acogimiento, Enfermaría, Urgencias médicas.

\section{INTRODUÇÃO}

A comunicação é um instrumento indispensável para um bom desenvolvimento em todas as áreas, contudo, para ser efetiva deve ter sua mensagem recebida com o mesmo sentido com o qual foi transmitida (SCHELLES, 2008). No âmbito da saúde a comunicação possui um papel fundamental, pois a partir desta, o profissional pode compreender o paciente de forma holística, para que o trabalho na assistência transcorra de modo efetivo e eficaz (BROCA PV, 2015).

Na comunidade surda o principal meio de comunicação se dá através da Língua de Sinais (LS), que apesar de conhecida em todo mundo, se apresenta com estrutura gramatical diferente em quase todos os países, podendo ter no mesmo país dois tipos de LS (PIZZIO AL, et al., 2010). A Língua Brasileira de Sinais (LIBRAS), foi oficializada no país no ano de 2002 através da Lei $n^{\circ} 10.436$ que surgiu frente à luta e conquista pelo direito igualitário dos surdos no país, como um meio legal para comunicação e expressão da população surda (BRASIL, 2002; TEDESCO JR e JUNGES JR, 2013).

É perceptível que a surdez perpassa um fator meramente médico, mediante diagnóstico. Uma vez que, para a pessoa surda, quando se trata de aspectos existenciais e sociais, existem uma conjuntura de fatores que implicam diretamente na sua inclusão ou não, dos quais o principal e mais básico é a comunicação. Fator esse que auxilia na inclusão do surdo na sociedade em todos os âmbitos, inclusive o de saúde (ESPOTE R, et al., 2013).

Conforme evidenciado no Decreto n5.626, artigo n²5 explanando sobre o Sistema Único de Saúde (SUS) e empresas que prestam assistência à saúde, para que se realize a inclusão plena do surdo, devem garantir a atenção integral à saúde nos diversos níveis de atenção, efetivando o acolhimento às pessoas surdas por profissionais capacitados e fluentes na sua devida língua, a LIBRAS, além de capacitar e auxiliar no processo de aprendizagem dos profissionais do SUS para uso da mesma, assim como a sua tradução e interpretação (BRASIL, 2005).

A fim de aprimorar e dar suporte ao decreto supracitado surgiu a Lei $n^{\circ} 13.146$ que assegura à pessoa com deficiência o acesso aos serviços de saúde, por meio de recursos tecnológicos e todas as formas de comunicação previstos nesta lei, além de assegurar a remoção de barreiras de comunicação e outras que venham interferir no acolhimento das pessoas com deficiência física, intelectual e mental (BRASIL, 2015).

Dentro do setor de saúde eventualmente existem momentos que fogem dos padrões aguardados na rotina de qualquer profissional, como por exemplo o encontro com um paciente surdo. Neste momento, as barreiras de comunicação não só dificultam a criação de vínculo com o paciente, mas podem implicar diretamente na 
qualidade do seu cuidado (CHAVEIRO N, et al., 2010). A relação profissional paciente com os níveis auditivos normais se estabelece através do código verbal, instrumento normalmente não utilizado por pessoas surdas que na sua grande maioria se comunica através da linguagem de sinais, desta forma, a ausência ou falha de comunicação inviabiliza um atendimento humanizado (CHAVEIRO N, et al., 2008; LESSA RTC, 2016).

A ausência de uso das LIBRAS pelos profissionais da saúde fragiliza a comunicação do deficiente auditivo, bem como seu acesso aos serviços de saúde em todos os níveis da assistência, prejudicando a qualidade do atendimento prestado. Portanto, torna-se indispensável que profissionais da saúde conheçam a LIBRAS, a fim de não comprometer o cuidado com o cliente (SILVA NAA, et al., 2020).

Diante do exposto, o objetivo deste estudo foi compreender a percepção de pessoas surdas sobre como tem sido seu acolhimento e cuidado em unidades de emergência por parte dos profissionais de enfermagem. A partir disso, se fez possível conhecer a história pregressa e atual destes surdos neste contexto de acolhimento e cuidado.

\section{MÉTODOS}

Caracterizou-se como estudo de pesquisa exploratória, com abordagem qualiquantitativa, realizado no contexto da percepção de pessoas surdas sobre o acolhimento e cuidado dos profissionais de enfermagem em unidades de emergência.

Contemplando e atendendo as normas e princípios éticos de pesquisas envolvendo seres humanos, guiadas pela Resolução 466/12 do CNS, e a fim de garantir o cumprimento da mesma, após autorização do serviço para realização da pesquisa e mediante parecer favorável do Comitê de Ética em Pesquisa da Universidade Estadual de Feira de Santana (UEFS) (CAAE: 35556520.1.0000.0053) os possíveis participantes foram contactados e voluntariamente aceitaram participar, assinando 0 Termo de Consentimento Livre e Esclarecido (TCLE).

Dez surdos residentes em Cachoeira-Ba, maiores de 18 anos, que já haviam sido atendidos anteriormente em alguma unidade de emergência, cumpriram com os critérios de inclusão e, portanto, participaram da pesquisa. Foram então excluídos os indivíduos surdos que não aceitaram participar do estudo, que não concordaram com o TCLE, ou indivíduos que não possuíam diagnóstico de surdez, que não eram residentes em Cachoeira-Ba, menores de idade, que não soubessem se comunicar em LIBRAS ou ainda aqueles que nunca haviam sido atendidos em um setor de emergência.

No processo da coleta de dados utilizou-se um roteiro semiestruturado com questões pertinentes à caracterização do indivíduo, tais como: sexo, idade, grau de instrução, nível de conhecimento de LIBRAS, atendimentos recebidos na unidade de emergência, dificuldades no processo do atendimento na unidade de emergência em virtude da barreira de comunicação. Na sequência, foram abordadas questões abertas referentes a: conhecimento conceitual sobre acolhimento e cuidado na unidade de emergência, experiências vividas neste ambiente, e a visão de cada indivíduo surdo sobre os profissionais de enfermagem que atuam no setor de emergência.

A entrevista foi realizada presencialmente e/ou virtualmente conforme disposição de cada participante surdo, após a assinatura e recolhimento do TCLE. A tradução se deu por intérpretes neutros em LIBRAS, e as respostas foram gravadas em vídeo por meio de um aparelho smartphone, para em seguida serem transcritas para a língua portuguesa pelas pesquisadoras do presente estudo. Todas as respostas foram transcritas e registradas na íntegra pelas pesquisadoras. Cabe salientar que as pesquisadoras são fluentes em LIBRAS, deste modo a análise do material se fez fidedigna. Para preservação da privacidade e anonimato de cada entrevistado, estes foram identificados por letras e números, sendo surdo 01 (S1), Surdo 02 (S2) e assim sucessivamente. As entrevistas foram realizadas entre 25 de outubro e 07 de dezembro de 2020.

Para análise das respostas apresentadas, as etapas subsequentes seguiram a abordagem de análise temática de conteúdo proposta por Bardin L (2011) primariamente se dando através da leitura flutuante, logo após, partindo para a escolha das categorias oriundas das questões norteadoras, e por fim promovendo a organização destas em indicadores ou temas. 


\section{RESULTADOS E DISCUSSÃO}

Através dos dados coletados foi possível obter o perfil demográfico dos participantes, estando representado por $75 \%$ de mulheres e apenas $25 \%$ de homens. De acordo com dados do IBGE (2010) coletados no mesmo lócus onde se deu a pesquisa, mais de $57 \%$ dos indivíduos surdos são homens, contudo pode-se justificar este resultado levando em consideração que as mulheres sempre são mais ativas no processo fazer saúde, desde a prevenção ao tratamento (TEXEIRA DBS, 2016), tornando-as sempre maioria dentro de pesquisas relacionadas à saúde.

O roteiro norteador utilizado facilitou a leitura do perfil de cada indivíduo surdo, culminando nos seguintes dados e percentuais, como: Grau de instrução (35\%) possuíam graduação de ensino superior; (35\%) graduação em andamento; (20\%) concluíram o ensino médio e (10\%) possuíam o ensino fundamental incompleto. Curso de LIBRAS: dos participantes apenas (20\%) relataram possuir, mesmo sendo dominantes/ fluentes na língua.

Percebeu-se então que os participantes da pesquisa eram em sua maioria já formados em nível superior, ou universitários. Tais achados reforçam os dados dos últimos anos, ressaltando certo crescimento no número de surdos inscritos nos cursos de formação acadêmica. O Censo da Educação Superior aponta que existem milhares de surdos matriculados nas instituições de ensino superior no Brasil (FREITAS DA, 2020).

O ápice deste estudo se deu a partir da compreensão das três questões direcionadas à temática do mesmo: O que você entende por acolhimento e cuidado na unidade de emergência? Em seu atendimento na unidade de emergência, como foi a experiência? E qual a sua visão sobre os profissionais de enfermagem que atuam na emergência?

Embasadas nessas questões norteadoras supramencionadas e na frequência das respostas, surgiram duas categorias, compostas respectivamente por três e duas subcategorias. A primeira tem por título acolhimento e cuidado na unidade de emergência e suas respectivas subcategorias são falta de empatia, acolhimento e cuidado; falta de conhecimento sobre o tema abordado e por fim visão sobre os profissionais de enfermagem que atuam na emergência. A categoria subsequente intitula-se vivencias do surdo quanto ao seu atendimento em unidade de emergência e suas subcategorias são respectivamente experiências vividas no ambiente de emergência pelos surdos com profissionais de enfermagem e falta de autonomia e independência.

\section{Acolhimento e cuidado na unidade de emergência}

A primeira categoria refere-se ao conhecimento e concepção dos indivíduos surdos sobre os cuidados e assistência de enfermagem na unidade de emergência. Correspondendo a primeira e a terceira questão do roteiro sobre as vivências do indivíduo neste ambiente e ao cuidado prestado pelo profissional de enfermagem. Esta categoria se dividiu em três subcategorias previamente citadas.

\section{Falta de empatia, acolhimento e cuidado}

Estes foram fatores muito presentes nos relatos dos participantes, evidenciando um certo conceito préestabelecido pelo fator cultural da comunidade surda devido à falta de comunicação, uma vez que em sua maioria para os indivíduos surdos isso significa falta de empatia e preconceito, culminando de forma geral em uma visão de total falta de acolhimento e cuidado da parte dos profissionais, conforme relatos:

"Não tem cuidado, os profissionais parecem não ter amor, apenas preconceito" (S1).

"O fator primordial para um bom acolhimento é comunicação, é a base de tudo, importantíssimo! Os profissionais precisam saber LIBRAS, para cuidar" (S11).

"É saber atender todos os tipos de pessoas, inclusive os surdos como eu" (S4).

O domínio e o bom uso da comunicação e fatores que a compõem, facilitam no processo do cuidar, tornando-se uma ferramenta indispensável para prestação de assistência (KAPPEL VB, et al., 2020). O encontro de um profissional de enfermagem que não sabe LIBRAS com o paciente surdo na emergência, 
evidencia as barreiras de comunicação, dificultando a criação de vínculo com o paciente, podendo implicar diretamente na qualidade do seu cuidado (CHAVEIRO N, et al., 2010).

\section{Falta de conhecimento sobre o tema abordado}

Percebeu-se através das falas trazidas a falta de conhecimento teórico sobre o assunto, uma vez que nem todos souberam ou conseguiram explicitar o que significa cuidado e acolhimento de um profissional da enfermagem, sobressaindo-se apenas a opinião particular, conforme falas a seguir:

"Não sei responder, nunca fui bem acolhida e cuidada nesses ambientes" (S5).

"Para mim, um bom atendimento é quando te explicam o que você tem ou os procedimentos que vão fazer, as medicações" (S7).

"É difícil falar sobre isso, porque o que desejamos nunca acontece, [...], se tem atenção para explicar o que está acontecendo, assim tem cuidado" (S8).

O indivíduo surdo tem dificuldade em conhecer algumas palavras em português, pois esta não é sua primeira língua, fazendo com que eles, em algumas situações confundam palavras, em especial palavras de escrita parecida, ou quando são pouco usadas no seu contexto de conversação (AVELAR TF, 2016).

Denota-se a partir dos relatos, ou da falta deles, que os participantes pouco sabem sobre saúde e seus direitos a ela, assim como desconhecem o que realmente significa acolhimento e cuidado partindo de um profissional de enfermagem. Essa realidade pode dar-se justamente pela falta de comunicação e informação de forma inclusiva nos diversos âmbitos da sociedade. Percebe-se que para a maioria dos surdos entrevistados "cuidado e acolhimento" significam, e estão atrelados, a ter empatia com a comunidade surda e saber LIBRAS.

\section{Visão sobre os profissionais de enfermagem que atuam na emergência}

Nesta subcategoria se retrata a opinião dos indivíduos surdos acerca dos profissionais de enfermagem no ambiente emergencial, baseando-se em visões pessoais e de experiências vividas, conforme relatos:

"Existem bons e maus profissionais como em todas as profissões, mas no caso da enfermagem especialmente na área da emergência é fundamental que os profissionais se capacitem para melhor atender a todos, especialmente aqueles que necessitam de cuidados especiais" (S3).

"Infelizmente eles não estão capacitados para atender a todos, vivi isso na pele durante minha gravidez, ainda precisa melhorar muito o cuidado e acolhimento" (S4).

"Para mim, por falta de comunicação acabam se tornando profissionais ruins. Eu quero poder ser atendido sozinho, ter autonomia, mas eles não são capazes" (S7).

"Ainda deixam a desejar pela falta de comunicação, sem contar que alguns nem se esforçam para dar atenção, não tem paciência, fazem parecer impossível atender um surdo" (S8).

Já que o paciente surdo possui uma diminuição ou perda total da capacidade de percepção de sons, seria necessário que o enfermeiro possuísse algum conhecimento básico de interpretação em LIBRAS, para assim, junto com o paciente levantar diagnósticos, identificar seus problemas e ajudar em seu processo de recuperação. Nesta relação terapêutica o enfermeiro tem a responsabilidade de interagir com o paciente impossibilitado de ouvir, para prestar acolhimento humanizado (SILVA PSS, 2014).

De fato, no momento do encontro entre o paciente surdo e o profissional de saúde, sentimentos como medo e insegurança adentram o profissional de saúde. Mas, tais sentimentos também invadem o paciente surdo, a ponto de impedir que este busque essa assistência com a mesma frequência que o paciente ouvinte (CHAVEIRO N, 2009).

Os relatos apresentados são carregados de um misto de sentimentos, uma vez que entre o desejo do paciente surdo e a realidade do acolhimento existe uma barreira conotada pela falta de comunicação. Embora 
a comunidade surda possua diversas conquistas elencadas no decorrer de sua trajetória, esta, é uma que ainda precisa ser alcançada. Ser incluído nos diversos ambientes da sociedade, especialmente naqueles que Ihes cabem por direito, é mais que uma necessidade, uma urgência, que pesa sobre os ombros dos mais diversos profissionais de saúde, neste caso especialmente, os enfermeiros que trabalham em unidades de emergência.

\section{Vivências do surdo quanto ao seu atendimento em unidade de emergência}

A segunda categoria alude às questões pessoais vividas por cada surdo no ato do acolhimento nas unidades de emergência. Fica explícita a necessidade de participação familiar nesse processo para resolução de problemas de baixa à alta complexidade. Esta categoria foi baseada na segunda questão do roteiro, e se dividiu em duas subcategorias.

\section{Experiências vividas no ambiente de emergência pelos surdos com profissionais de enfermagem}

"[...] O problema é que os profissionais nunca falam comigo ou para mim, apenas com minha mãe, eu fico "boiando" até ela me explicar algo. Isso é constrangedor! Além disso, às vezes não consigo compreender quando escrevem" (S11).

"Estava muito mal, sentia várias cólicas, chamei minha mãe pois não queria ir só, por causa da comunicação, mas ela não quis ir, não deu importância, eu fui ficando cada vez pior e mais nervosa, quando decidi ir. Chegando lá ninguém sabia me atender, até que chamaram um rapaz que era conhecido meu, e ele verificou minha pressão, tentou me acalmar, mas foi horrível, ninguém me entendia" (S1).

"Sempre fui sozinho, me viro, uso mímicas, escrevo, mostro no celular, mas é muito difícil os profissionais não saberem minha língua. Na hora das medicações tenho sempre medo, de não entenderem se for algo que tenho alergia ou de não explicarem" (S10).

"O atendimento é horrível, não tem inclusão nenhuma" (S6).

Grande parte dos profissionais não têm domínio da LIBRAS, utilizando-se de outros meios, como a linguagem escrita ou a leitura labial, que nem sempre contemplam o indivíduo surdo (OLIVEIRA YCA, et al., 2015). Na maioria das vezes o profissional da saúde faz uso da comunicação de forma não verbal, expressada através de mímicas, caretas e as mais variadas formas que se possa imaginar a fim de promover uma comunicação "parecida" com a LIBRAS, porém isso faz com que o profissional se comporte de maneira distante e indiferente, gerando desconfiança e afetando a comunicação (KAPPEL, et al. 2020).

Tais fatos são confirmados em cada detalhe das falas expressas, diferente do que está preconizado sobre os direitos e deveres sobre a saúde, e a seguridade de cada indivíduo, os pacientes surdos não se sentem acolhidos, cuidados e muito menos satisfeitos com o atendimento que recebem em unidades de emergência, provocando na maioria dos indivíduos constrangimento, insegurança e medo. Além de tudo isso, se faz necessário expor o grande risco a própria saúde e vida desses indivíduos, afinal de contas a falta de comunicação ou compreensão dos fatos pode facilmente culminar em erros brandos ou até mesmo fatais, como explicitado nos relatos.

\section{Falta de autonomia e independência}

A subcategoria dois, evidencia-se pelos relatos de total dependência de se ter um familiar ou terceiro durante e no processo da ida ao ambiente emergencial, ocasionando, conforme expressado, pouca ou nenhuma autonomia, assim como constrangimento e falta de privacidade em diversas situações, tais como relatadas abaixo:

"Esse ano eu ganhei meu primeiro filho, e na hora de ir para emergência foi muito difícil, [...]como todos precisavam usar máscara se quer leitura labial eu conseguia fazer, senti muito medo e insegurança. Várias vezes a equipe tentou se comunicar comigo e não teve sucesso, precisavam chamar o meu esposo e falar com ele apenas. Foi muito triste para mim" (S4). 
"Há alguns anos sofri um acidente de trabalho, minha mão ficou presa em uma máquina de corte de couro, tinha muito sangue e quase perdi a mão, meu patrão e uma enfermeira da empresa tiveram que me levar até a casa da minha mãe pra ela me acompanhar no hospital e conversar com a equipe, ela sempre é minha voz, não sabe LIBRAS mas me entende como pode e sempre fala o que sinto, sempre foi assim, no posto, hospital e nessa emergência também, eles falam tudo pra ela, porque não sabem LIBRAS e nunca tem comunicação comigo, sempre fico perdida sem saber o que está acontecendo" (S2).

"Sempre que preciso, tenho que chamar minha mãe para ir comigo, porque eles não me entendem, não tem comunicação. Mesmo ela estando cansada ou ocupada, só tem ela. Fico pensando o que vou fazer quando ela não estiver mais aqui" (S7).

"Sempre vou acompanhada da minha mãe, [...] sem intérprete não consigo expressar o que estou sentindo, e tenho medo, por falta de comunicação podem me dar uma medicação errada, ou fazer um procedimento errado" (S9).

Para que essa barreira de comunicação entre profissionais e pacientes diminua, muitas vezes a utilização de uma terceira pessoa no processo assistencial é indispensável. Essa terceira pessoa é o profissional intérprete ou um familiar, mas o fator "perda da privacidade" deve ser levado em consideração (OLIVEIRA YCA, et al., 2015).

Além de tudo, observa-se a dificuldade de encontrar profissionais intérpretes para o acompanhamento na assistência em saúde, recaindo sobre a família do paciente surdo a responsabilidade. Entretanto, esta interlocução não contribui para uma inclusão efetiva e proativa do surdo em seu atendimento, tampouco endossa seu protagonismo neste processo. Os surdos valorizam o intérprete, porém, a confiança, a vergonha de se expor frente ao intérprete e os sentimentos de pena externados pelo mesmo, são fatores que causam um bloqueio para o surdo (CHAVEIRO N, 2005).

Autonomia e independência, são características constantemente prezadas no ensino de pais para filhos, assim como nos âmbitos escolares e sociais. Contudo, há tamanha implicância e limitação quando se trata do indivíduo surdo, deveras superprotegido pelos familiares, desassistido e muitas vezes até esquecido pela sociedade e governo. Resultando em cidadãos inseguros, dependentes, e às vezes até incapazes de desempenharem seus direitos e deveres. Ora por consequência da falta de autonomia, ora por ineficiência e despreparo das instituições e órgãos, como se pôde notar.

Estudos como este servem justamente para abrir os olhos, alertar e esclarecer assuntos e realidades vividas, pouco notáveis ou consideradas pelas esferas governamentais, familiares, profissionais e até mesmo individuais; a fim de promover mudanças positivas, crescimento social, qualidade de vida, inclusão e equidade.

\section{CONCLUSÃO}

Diante do exposto percebeu-se a urgência de fazer saúde com mais inclusão para o paciente surdo. De acordo com relatos dos vários indivíduos, existe pouco ou nenhum domínio da LIBRAS por parte dos profissionais de enfermagem, dificultando o atendimento holístico para pessoas com surdez na emergência hospitalar. Dessa forma, suscita-se então, a necessidade do aprendizado dessa língua para seguridade da comunicação.

\section{REFERÊNCIAS}

1. AVELAR TF, FREITAS KPS. A importância do português como segunda língua na formação do aluno surdo. Revista sinalizar, 2016; 1(1):12-24.

2. BARDIN L. Análise de conteúdo. Trad. Luís A e Augusto P. 5 ed. Lisboa: edições 70 LTDA, 2011. 
3. BRASIL. Decreto $n^{\circ} 5.626$, de 22 de dezembro de 2005. 2005 . Disponível em: https://www2.camara.leg.br/legin/fed/decret/2005/decreto-5626-22-dezembro-2005-539842-publicacaooriginal39399-pe.html. Acesso em 15 de abril de 2020.

4. BROCA, PV, FERREIRA MA. Processo de comunicação na equipe de enfermagem fundamentado no diálogo entre Berlo e King. Escola Anna Nery revista de enfermagem, 2015; 19(3): 467-474.

5. CHAVEIRO N, BARBOSA MA. Assistência ao surdo na área de saúde como fator de inclusão social. Revista da Escola de Enfermagem da USP, 2005; 39(4): 417-22.

6. CHAVEIRO N, et al. Atendimento à pessoa surda que utiliza a língua de sinais, na perspectiva do profissional da saúde. Cogitare enfermagem, 2010; 15(4): 639-45.

7. CHAVEIRO N, et al. Relação do paciente surdo com o médico. Revista Brasileira de Otorrinolaringologia, 2009; 75(1): 147-50.

8. CHAVEIRO N, et al. Revisão de literatura sobre o atendimento ao paciente surdo pelos profissionais da saúde. Rev. esc. enferm., 2008, 42(3): 578-583.

9. ESPOTE R, et al. Inclusão de surdos: revisão integrativa da literatura científica. Psico USF, 2013; 18(1)77-88.

10. FREITAS DA, EULÁLIO WES. Surdos e o Ensino Superior no Brasil: uma reflexão. Revista Eletrônica Nacional de Educação Física, 2020; 10(15):42 - 65.

11. INSTIRUTO BRASILEIRO DE GEOGRAFIA E ESTATÍSTICA (IBGE). Disponível em: https://www.ibge.gov.br/apps/snig/v1/?loc=0\&cat=-1,-2,-3,128\&ind=4643>. Acesso em 15 de abril de 2020.

12. KAPPEL VB, et al. Comunicação profissional família em um centro de atenção psicossocial infanto-juvenil: facilidades e dificuldades. Texto e Contexto Enfermagem, 2020; 29(20): 1-15.

13. LESSA RTC, ANDRADE EGS Libras e o atendimento ao cliente surdo no âmbito da saúde. Revista cient. Sena Aires, 2016; 5(2):95-104.

14. OLIVEIRA YCA, et al. Comunicação como ferramenta essencial para assistência à saúde dos surdos. Revista de Saúde Coletiva, 2015; 25(1):307-320.

15. PIZZIO AL, et al. Língua brasileira de sinais VI. Editora UFSC., 2010;1-67.

16. SCHELLES S. A importância da linguagem não-verbal nas relações de liderança nas organizações. Revista esfera, 2008; 06(1): 1-8.

17. SILVA NAA, et al. A perspectiva do surdo enquanto paciente no atendimento à saúde. Única Cadernos Acadêmicos, 2020; 3(1):6.

18. SILVA PSS, et al. A enfermagem e a utilização da língua brasileira de sinais no atendimento ao deficiente auditivo. Revista Uningá Review, 2014; 17(1): 05-12.

19. TEDESCO JR, JUNGES JR. Desafios da prática do acolhimento de surdos na atenção primária. Revista de saúde pública, Rio de Janeiro, 2013; 29(8): 1685-1689.

20. TEXEIRA DBS, CRUZ SPL. Atenção à saúde do homem: análise da sua resistência na procura dos serviços de saúde. Revista cubana de enfermería, 2016; 32(4): 1-04. 